\title{
Very boosted Higgs in gluon fusion
}

\author{
C. Grojean, ${ }^{a}$ E. Salvioni, ${ }^{b, c, d}$ M. Schlaffer ${ }^{e}$ and A. Weiler ${ }^{c, e}$ \\ ${ }^{a}$ ICREA at IFAE, Universitat Autónoma de Barcelona, \\ E-08193 Bellaterra, Spain \\ ${ }^{b}$ Department of Physics, University of California, \\ Davis, CA 95616, USA \\ ${ }^{c}$ Theory Division, Physics Department, CERN, \\ CH-1211 Geneva 23, Switzerland \\ ${ }^{d}$ Dipartimento di Fisica e Astronomia, Università di Padova and INFN, Sezione di Padova, \\ Via Marzolo 8, I-35131 Padova, Italy \\ e DESY, \\ Notkestrasse 85, D-22607 Hamburg, Germany \\ E-mail: christophe.grojean@cern.ch, esalvioni@ucdavis.edu, \\ matthias.schlaffer@desy.de, andreas.weiler@cern.ch
}

AbstRACT: The Higgs production and decay rates offer a new way to probe new physics beyond the Standard Model. While dynamics aiming at alleviating the hierarchy problem generically predict deviations in the Higgs rates, the current experimental analyses cannot resolve the long- and short-distance contributions to the gluon fusion process and thus cannot access directly the coupling between the Higgs and the top quark. We investigate the production of a boosted Higgs in association with a high-transverse momentum jet as an alternative to the $t \bar{t} h$ channel to pin down this crucial coupling. Presented first in the context of an effective field theory, our analysis is then applied to models of partial compositeness at the $\mathrm{TeV}$ scale and of natural supersymmetry.

Keywords: Higgs Physics, Beyond Standard Model

ArXiv EPRINT: 1312.3317 


\section{Contents}

1 Introduction 1

2 Analysis of $p p \rightarrow h+$ jet $\quad 4$

2.1 Breaking the degeneracy 6

2.2 Limitations and possible improvements 8

3 Resolving the top partner spectrum in composite Higgs models $\quad 8$

$\begin{array}{lll}3.1 & \text { A simple explicit model } & 10\end{array}$

$\begin{array}{ll}3.2 \text { Validity of the effective theory approach } & 11\end{array}$

4 Resolving the stop spectrum/mixing in supersymmetric models $\quad 12$

5 Conclusions and outlook $\quad 14$

A Amplitudes for $p p \rightarrow h+$ jet with $C P$-violating couplings $\quad 16$

\section{Introduction}

With the discovery $[1,2]$ last year of a new resonance, whose properties so far show no deviations from those of the long sought-after Higgs boson, a new era started in the understanding of the Standard Model (SM) of particle physics. In the absence of any evidence for any other new degree of freedom at the weak scale, a mass gap is likely to separate the SM particles from the dynamics generating and stabilizing the Higgs potential. Our ignorance about the new physics sector can thus be conveniently parametrized in terms of a set of higher dimensional operators built out of SM fields obeying the SM symmetries. For a single family of fermions, there are 59 independent ways to deform the SM [3, 4]. Of particular interest are the 30 deformation directions affecting Higgs physics [5-7]. Actually, 20 of them were already constrained at the per-mil/per cent level before the Higgs discovery itself, thanks to electroweak measurements involving massive gauge bosons and bounds on quark and lepton dipole moments. The Higgs data collected by ATLAS and CMS (as well as by the Tevatron experiments) start putting interesting bounds on the remaining $8 C P$ even and $2 C P$-odd directions $[7,8]$. In this regard, a Higgs mass around $125 \mathrm{GeV}$ offers remarkable opportunities to probe these directions, since it opens numerous decay channels with a rate accessible with the current integrated luminosity delivered by the LHC. 
Among these operators, four are particularly important, since they control the main production mechanism of the Higgs, namely gluon fusion. These are ${ }^{1}$

$$
\begin{aligned}
\mathcal{O}_{y} & =\frac{y_{t}}{v^{2}}|H|^{2} \bar{Q}_{L} \tilde{H} t_{R}, & \mathcal{O}_{H} & =\frac{1}{2 v^{2}} \partial_{\mu}|H|^{2} \partial^{\mu}|H|^{2}, \\
\mathcal{O}_{g} & =\frac{\alpha_{s}}{12 \pi v^{2}}|H|^{2} G_{\mu \nu}^{a} G^{a \mu \nu}, & \widetilde{\mathcal{O}}_{g} & =\frac{\alpha_{s}}{8 \pi v^{2}}|H|^{2} G_{\mu \nu}^{a} \widetilde{G}^{a \mu \nu},
\end{aligned}
$$

added to the SM as

$$
\mathcal{L}_{\text {eff }}=\mathcal{L}_{\mathrm{SM}}+\left(c_{y} \mathcal{O}_{y}+\text { h.c. }\right)+c_{H} \mathcal{O}_{H}+c_{g} \mathcal{O}_{g}+\tilde{c}_{g} \widetilde{\mathcal{O}}_{g}
$$

From this Lagrangian we can extract the terms relevant to Higgs production in gluon fusion, ${ }^{2}$

$$
-\kappa_{t} \frac{m_{t}}{v} \bar{t} t h+\kappa_{g} \frac{\alpha_{s}}{12 \pi} \frac{h}{v} G_{\mu \nu}^{a} G^{\mu \nu a}+i \tilde{\kappa}_{t} \frac{m_{t}}{v} \bar{t} \gamma_{5} t h+\tilde{\kappa}_{g} \frac{\alpha_{s}}{8 \pi} \frac{h}{v} G_{\mu \nu}^{a} \widetilde{G}^{a \mu \nu}+\mathcal{L}_{\mathrm{QCD}}
$$

where at linear order in the coefficients multiplying the operators in eq. (1.2) we find

$$
\kappa_{t}=1-\operatorname{Re}\left(c_{y}\right)-\frac{c_{H}}{2}, \quad \kappa_{g}=c_{g}, \quad \tilde{\kappa}_{t}=\operatorname{Im}\left(c_{y}\right), \quad \tilde{\kappa}_{g}=\tilde{c}_{g}
$$

The first two terms in eq. (1.3) are invariant under $C P$, whereas the third and fourth terms violate $C P$. We will be mainly interested in $C P$-conserving effects, therefore we set $\tilde{\kappa}_{t}=\tilde{\kappa}_{g}=0$ in the following. We will however return briefly to the $C P$-odd case later (notice that since there is no interference between the $C P$-even and -odd amplitudes for $p p \rightarrow h+$ jet, the two cases can be discussed separately). The coefficient $\kappa_{t}$ corrects the top Yukawa coupling, which controls the top loop contribution to $g g \rightarrow h$ production, whereas the coefficient $\kappa_{g}$ gives a direct contribution to this process. Unfortunately, the lightness of the Higgs boson plays a malicious role and makes it impossible to disentangle shortand long-distance contribution to the gluon fusion total rate. This limitation is embodied in the Higgs low energy theorem $[10,11]$ that ensures that the inclusive production cross section is proportional to $\left(\kappa_{t}+\kappa_{g}\right)^{2}$. In fact, from eq. (1.3) we readily obtain

$$
\frac{\sigma_{\text {incl }}\left(\kappa_{t}, \kappa_{g}\right)}{\sigma_{\text {incl }}^{\text {SM }}} \simeq\left(\kappa_{t}+\kappa_{g}\right)^{2}\left(1-\frac{7}{15} \frac{\kappa_{g}}{\kappa_{t}+\kappa_{g}} \frac{m_{h}^{2}}{4 m_{t}^{2}}\right) \simeq\left(\kappa_{t}+\kappa_{g}\right)^{2},
$$

where in the second equality we neglected corrections that will remain untouchable at the LHC [12]. The only hope then to have a direct access to the top Yukawa coupling is through the $p p \rightarrow t \bar{t} h$ process, which is notoriously difficult due to its high threshold, its small rate and its complicated final state with copious decay products. Exploratory studies concluded that the sensitivity in the measurement of the top Yukawa coupling through the

\footnotetext{
${ }^{1} G_{\mu \nu}^{a}$ denotes the QCD gauge-field strength and $\widetilde{G}^{a \mu \nu}=(1 / 2) \epsilon^{\mu \nu \rho \sigma} G_{\rho \sigma}^{a}$ its dual. $\alpha_{s}$ is the QCD coupling strength. $H$ is the SM Higgs doublet and $\tilde{H} \equiv i \sigma_{2} H^{*}, v$ is the SM Higgs vacuum expectation value related to the Fermi constant by $v=\left(\sqrt{2} G_{F}\right)^{-1 / 2} \simeq 246 \mathrm{GeV}, y_{t}$ is the SM top Yukawa coupling, and finally $Q_{L}$ and $t_{R}$ are the $\mathrm{SU}(2)_{L}$ quark doublet and charge-2/3 quark singlet of the third generation.

${ }^{2}$ See ref. [9] for a study of the effects of Higgs-gluons interactions of dimension 7 in $h+$ jet and $h+2$ jets observables.
} 
$t \bar{t} h$ process will be limited to about $10 \%$ within the LHC high-luminosity program $[13,14]$ and Higgs factories like a 500-GeV ILC or a $80 / 100-\mathrm{km}$ TLEP will be needed to bring this sensitivity down to a few per-cent level $[15,16]$.

In this paper we propose an alternative way to break the degeneracy between the two coefficients $\kappa_{t}$ and $\kappa_{g}$ and thus to provide direct information on the top Yukawa coupling. Our idea relies on the use of extra radiation in the $g g \rightarrow h$ process that will allow us to explore the structure of the top loop. When the extra radiation carries away a large amount of energy and boosts the Higgs boson, the process effectively probes the ultraviolet structure of the top loop. Notice that the extra radiation cannot be in the form of a photon, as the amplitude for $g g \rightarrow h+\gamma$ vanishes due to Furry's theorem. One is therefore led to consider the production of $h$ in association with a jet. ${ }^{3}$

Resolving the degeneracy between the two contributions to gluon-fusion Higgs production is not a mere academic exercise. There exist some new physics scenarios whose identification is endangered by the limitation of the LHC to separately measure $\kappa_{t}$ and $\kappa_{g}$. The prime examples are composite Higgs models: the top sector is enlarged and encompasses extra heavy vector-like fermions that mix with the SM top quark. These top partners have a double effect to simultaneously shift the top Yuakwa coupling and to also give rise to a contact interaction between the gluons and the Higgs boson. As it was first noticed in ref. [19] and later explored in refs. [20-23], these two effects precisely annihilate in the $g g \rightarrow h$ process in minimal (and most popular) models. Another cancellation can occur in natural supersymmetric (SUSY) scenarios, when a large mixing between the stops conspires to nullify the total stop contribution to $g g \rightarrow h$. Even though this is a particular region in the SUSY parameter space, an accumulation of experimental hints might point to such a situation and some experimental ingenuity will be required to directly see the low-lying stops [24]. Thus new physics might well be present in the data already collected at the LHC, while the measurements performed up to now remain blind to it. We advocate that the $p p \rightarrow h+$ jet channel can be competitive or at least complementary with the more studied $p p \rightarrow t \bar{t} h$ channel to unveil hidden new physics. For previous studies addressing the use of the Higgs transverse momentum distribution as a handle on New Physics, see for example refs. [25-27].

This paper is organized as follows. In section 2 we present our analysis of the $p p \rightarrow$ $h+$ jet process, by adopting an effective theory approach to describe the effects of heavy new physics. In order to obtain an estimate of the LHC potential to disentangle the current degeneracy in the plane of effective couplings, we focus on the promising channel where the Higgs boson decays to two collimated tau leptons. Section 3 studies the ability of the boosted Higgs regime to probe the spectrum of top partners in composite Higgs models, whereas section 4 looks at the $h+$ jet process as a way to probe light stops in supersymmetric extensions of the SM. Finally, section 5 collects our conclusions. We also include an appendix, where formulae for the $p p \rightarrow h+$ jet amplitudes mediated by $C P$ violating couplings are reported.

\footnotetext{
${ }^{3}$ The process $g g \rightarrow h Z$ is also interesting, despite being subleading with respect to the tree-level contribution from $q \bar{q} \rightarrow h Z$. See refs. [17, 18] for very recent studies.
} 

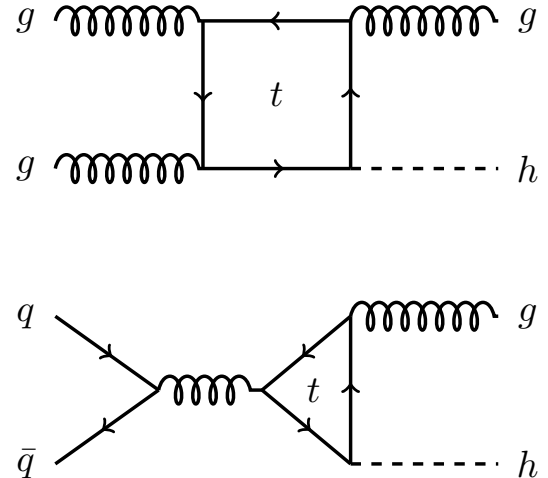
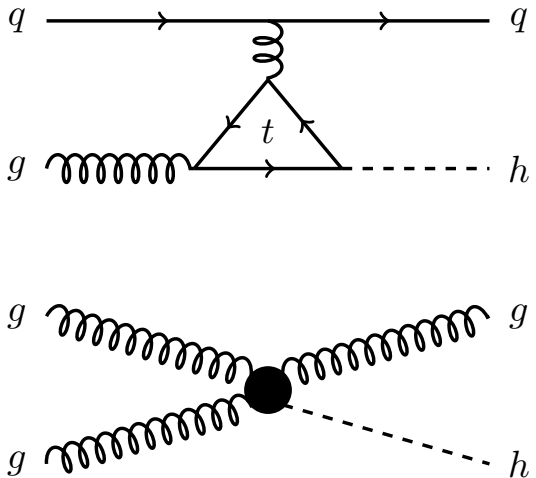

Figure 1. Example Feynman diagrams for $p p \rightarrow h+$ jet in the SM and with the contact term.

\section{Analysis of $p p \rightarrow h+$ jet}

At the parton level, three subprocesses contribute to the $p p \rightarrow h+$ jet cross section: these are $g g, q g, q \bar{q} \rightarrow h+$ jet. $^{4}$ The expressions of the SM matrix elements for $g g \rightarrow h g$ and $q \bar{q} \rightarrow h g$, mediated by quark loops, were first calculated at LO in QCD in ref. [28] and shortly after with a different notation in ref. [29], which we used for our calculations. The matrix element for the $q g \rightarrow h q$ process is obtained from the one of $q \bar{q} \rightarrow h g$ by crossing. Some of the Feynman diagrams contributing to $p p \rightarrow h+$ jet are shown in figure 1 . When the Lagrangian in eq. (1.3) is considered, the top contribution to the amplitudes is simply given by the SM one rescaled by the modified coupling $\kappa_{t} .{ }^{5}$ On the other hand, the contribution of heavy top partners in the loop is described by the effective interaction parameterized by $\kappa_{g}$, which generates Feynman diagrams such as the lower-right one in figure 1. Roughly speaking, this description is reliable as long as the mass of the heavy states is larger than the transverse momentum cut applied, see section 3 for a more precise assessment. The corresponding matrix element is obtained from the SM one by sending to infinity the mass of the quark running in the loop. Thus the matrix element squared for each partonic subprocess can be written as

$$
|\mathcal{M}|^{2} \propto\left|\kappa_{t} \mathcal{M}_{\mathrm{IR}}\left(m_{t}\right)+\kappa_{g} \mathcal{M}_{\mathrm{UV}}\right|^{2}
$$

where $\mathcal{M}_{\mathrm{IR}}$ denotes the amplitude mediated by top loops, and $\mathcal{M}_{\mathrm{UV}}$ the amplitude mediated by the effective point-like interaction. It follows that the hadronic cross section for $p p \rightarrow h+$ jet can be written as a quadratic polynomial in $\kappa_{t}$ and $\kappa_{g}$. Given a transverse momentum cut $p_{T}^{\min }$ and summing over all partonic subprocesses, we can write

$$
\frac{\sigma_{p_{T}^{\min }}\left(\kappa_{t}, \kappa_{g}\right)}{\sigma_{p_{T}^{\min }}^{\mathrm{SM}}}=\left(\kappa_{t}+\kappa_{g}\right)^{2}+\delta \kappa_{t} \kappa_{g}+\epsilon \kappa_{g}^{2}
$$

\footnotetext{
${ }^{4}$ For brevity, we denote the sum $q g+\bar{q} g$ by $q g$.

${ }^{5}$ In the SM, the effect of including the bottom quark contribution in addition to the dominant one due to the top is only of a few percent, if the cut on the transverse momentum is larger than $50 \mathrm{GeV}[27,30,31]$. Since we are interested in larger Higgs transverse momenta, we consistently neglect the bottom in our calculation.
} 


\begin{tabular}{|c|r|r|r|r|r|r|r|r|}
\hline$\sqrt{s}[\mathrm{TeV}]$ & $p_{T}^{\min }[\mathrm{GeV}]$ & $\sigma_{p_{T}^{\text {min }}}^{\mathrm{SM}}[\mathrm{fb}]$ & \multicolumn{1}{c|}{$\delta$} & \multicolumn{1}{c|}{$\epsilon$} & $g g, q g[\%]$ & $\tilde{\gamma} \cdot 10^{2}$ & \multicolumn{1}{c}{$\tilde{\delta}$} & \multicolumn{1}{c|}{$\tilde{\epsilon}$} \\
\hline \multirow{6}{*}{} & 100 & 2180 & 0.0031 & 0.031 & 67,31 & 2.6 & 0.033 & 0.031 \\
& 150 & 837 & 0.070 & 0.13 & 66,32 & 1.7 & 0.094 & 0.13 \\
& 200 & 351 & 0.20 & 0.30 & 65,34 & 0.28 & 0.22 & 0.30 \\
& 250 & 157 & 0.39 & 0.56 & 63,36 & 0.20 & 0.41 & 0.56 \\
& 300 & 74.9 & 0.61 & 0.89 & 61,38 & 1.0 & 0.64 & 0.89 \\
& 350 & 37.7 & 0.85 & 1.3 & 58,41 & 2.2 & 0.91 & 1.3 \\
& 400 & 19.9 & 1.1 & 1.7 & 56,43 & 3.4 & 1.2 & 1.7 \\
& 450 & 10.9 & 1.4 & 2.3 & 54,45 & 4.6 & 1.5 & 2.3 \\
& 500 & 6.24 & 1.7 & 2.9 & 52,47 & 5.6 & 1.8 & 2.9 \\
& 550 & 3.68 & 2.0 & 3.6 & 50,49 & 6.5 & 2.2 & 3.6 \\
& 600 & 2.22 & 2.3 & 4.4 & 48,51 & 7.3 & 2.5 & 4.4 \\
& 650 & 1.38 & 2.6 & 5.2 & 46,53 & 7.9 & 2.9 & 5.2 \\
& 700 & 0.871 & 3.0 & 6.2 & 45,54 & 8.4 & 3.2 & 6.2 \\
& 750 & 0.562 & 3.3 & 7.2 & 43,56 & 8.8 & 3.6 & 7.2 \\
& 800 & 0.368 & 3.7 & 8.4 & 42,57 & 9.1 & 3.9 & 8.4 \\
\hline \multirow{6}{*}{100} & 500 & 964 & 1.8 & 3.1 & 72,28 & 5.0 & 1.9 & 3.1 \\
& 2000 & 1.01 & 14 & 78 & 56,43 & 7.0 & 15 & 78 \\
\hline
\end{tabular}

Table 1. Summary table of the cross sections for $p p \rightarrow h+$ jet at proton-proton colliders with $\sqrt{s}=14 \mathrm{TeV}$ and $\sqrt{s}=100 \mathrm{TeV}$. The third, fourth and fifth column show, for the given cut on $p_{T}>p_{T}^{\min }$, the parameters of the semi-numerical formula in eq. (2.2). The sixth column shows the fraction of the SM cross section coming from the partonic subprocesses $g g$ and $q g$ (the contribution of the $q \bar{q}$ channel is always smaller than $2 \%$ ). The last three columns show the values of the parameters of the semi-numerical formula for the $C P$-odd cross sections, eq. (2.3).

where $\sigma$ is the cross section for $p p \rightarrow h+$ jet and the numerical coefficients $\{\delta, \epsilon\}$ depend on $p_{T}^{\min }$. Their values are listed in table 1 for an LHC center of mass energy of $\sqrt{s}=14 \mathrm{TeV}$ and various choices of $p_{T}^{\min }$. In the calculation, we set the factorization and renormalization scales to the Higgs transverse mass, $\mu_{\text {fact }}=\mu_{\text {ren }}=m_{T}(h)=\sqrt{m_{h}^{2}+p_{T}^{2}}$, where $m_{h}$ is the mass of the Higgs and $p_{T}^{2}=\hat{t} \hat{u} / \hat{s}$ its transverse momentum squared. The calculation of the strong coupling constant and the convolution with the PDFs was done using the MSTW 2008 LO PDFs [32]. The values of the scalar integrals were obtained from LoopTools2.8 [33]. We have compared our results for the SM cross sections with MCFM-6.6 [34] and with HIGLU [35], finding exact agreement. In table 1 we also show the values of $\{\delta, \epsilon\}$ for a $100 \mathrm{TeV}$ proton-proton collider, for two choices of $p_{T}^{\min }$, to illustrate the possibilities of such a machine. However, since the efficiencies achievable at the very-high-energy collider are not known yet, we do not consider these values further in our analysis. We note that the $g g$ and $q g$ initial states contribute to the total cross section roughly at the same level, with the relative contribution of $q g$ increasing at higher $p_{T}$. The $q \bar{q}$ initial state contributes to the total cross section at the level of $1 \div 2 \%$.

It is interesting to consider also the possibility that the gluon fusion process receives contribution from $C P$-violating new physics. Considering the operators parameterized by 
$\tilde{\kappa}_{t}$ and $\tilde{\kappa}_{g}$ in eq. (1.3), we can write the cross section for $p p \rightarrow h+$ jet in a form similar to eq. $(2.2)$

$$
\frac{\sigma_{p_{T}^{\min }}\left(\tilde{\kappa}_{t}, \tilde{\kappa}_{g}\right)}{\sigma_{p_{T}^{\min }}^{\operatorname{SM}}}=\frac{9}{4}\left[\left(\tilde{\kappa}_{t}+\tilde{\kappa}_{g}\right)^{2}+\tilde{\gamma} \tilde{\kappa}_{t}^{2}+\tilde{\delta} \tilde{\kappa}_{t} \tilde{\kappa}_{g}+\tilde{\epsilon} \tilde{\kappa}_{g}^{2}\right]
$$

where the numerical coefficients $\{\tilde{\gamma}, \tilde{\delta}, \tilde{\epsilon}\}$ depend on $p_{T}^{\min }$. Their values are listed in table 1 , for the same parameter choices we made in the $C P$-conserving case, and were calculated by using the analytical results for the $p p \rightarrow h+$ jet cross section mediated by $C P$-violating couplings, reported in the appendix. Notice that the overall factor of $9 / 4$ appearing in eq. (2.3) is chosen in accordance with the expression of the inclusive cross section, which reads $\sigma_{\text {incl }}\left(\tilde{\kappa}_{t}, \tilde{\kappa}_{g}\right) / \sigma_{\text {incl }}^{\mathrm{SM}} \simeq 9\left(\tilde{\kappa}_{t}+\tilde{\kappa}_{g}\right)^{2} / 4$.

It has been shown [36] that the effective operators $h G_{\mu \nu}^{a} G^{a \mu \nu}$ and $h G_{\mu \nu}^{a} \widetilde{G}^{a \mu \nu}$, when added in turn and with the same normalization to the QCD Lagrangian, lead to identical amplitudes squared for the processes $g g, q g, q \bar{q} \rightarrow h+$ jet. This implies that the identity $\epsilon=\tilde{\epsilon}$ holds for any value of $p_{T}^{\min }$, as can be seen in table 1 .

\subsection{Breaking the degeneracy}

We now aim at giving an estimate of the potential of the boosted Higgs measurement to resolve the ambiguity in the plane of the couplings $\left(\kappa_{t}, \kappa_{g}\right)$. While a full analysis would ideally combine several decay modes of the Higgs, a first estimate can be obtained by looking at one channel only. Because, as shown in table 1, in order to break the degeneracy we need to consider very large Higgs transverse momenta and therefore small rates, it is natural to consider first the decay channels with the largest branching ratios, namely $h \rightarrow b \bar{b}, W W, \tau \tau$. Here we focus on the last mode, and we will comment briefly on other possibilities at the end of this section. For a Higgs transverse momentum larger than $500 \mathrm{GeV}$, the typical angular separation between the two taus is $\Delta R \sim 2 m_{h} / p_{T} \lesssim 0.5$. As a consequence, when at least one of the taus decays hadronically, the standard tau-tagging techniques will fail, due to the non-isolation of the hadronic tau candidate(s). However, such 'ditaujets' can be tagged by adapting the usual tau-tagging algorithm, as suggested in ref. [37], whose efficiencies for signal identification are assumed here. ${ }^{6}$ Including the Higgs and tau branching ratios, we obtain the following estimate of the total efficiency

$$
\epsilon_{\mathrm{tot}}=\operatorname{BR}(h \rightarrow \tau \tau) \times\left(\sum_{i=\tau_{\ell} \tau_{\ell}, \tau_{\ell} \tau_{h}, \tau_{h} \tau_{h}} \operatorname{BR}(\tau \tau \rightarrow i) \epsilon_{i}\right) \simeq 2 \times 10^{-2}
$$

where we assumed the $\mathrm{SM}$ value for $\mathrm{BR}(h \rightarrow \tau \tau)$ [38].

To break the degeneracy in the $\left(\kappa_{t}, \kappa_{g}\right)$ plane that plagues inclusive Higgs production, we need to combine the measurements of both the inclusive and boosted rates. On the one

\footnotetext{
${ }^{6}$ Reference [37] applied ditau-tagging to the case of a $Z^{\prime}$ decaying to $Z h$. We make use of the efficiencies reported in their table I for a $2 \mathrm{TeV} Z^{\prime}$, which gives a Higgs $p_{T}$ roughly similar to the case we are considering. We assume efficiencies that include in addition to the ditau-jet tagging also the reconstruction of the Higgs mass peak, as it seems unavoidable that an experimental analysis would need to exploit that information.
} 


\begin{tabular}{|c|c|c|c|c|c|}
\hline$\mu=\mu_{\text {ren }}=\mu_{\mathrm{fac}}$ & $\sigma_{150 \mathrm{GeV}}[\mathrm{fb}]$ & $K_{150 \mathrm{GeV}}$ & $\sigma_{650 \mathrm{GeV}}[\mathrm{fb}]$ & $K_{650 \mathrm{GeV}}$ & $\mathcal{R}$ \\
\hline$m_{T} / 2$ & $1.2 \cdot 10^{3}$ & 1.16 & 2.0 & 1.14 & $1.66 \cdot 10^{-3}$ \\
\hline$m_{T}$ & $0.83 \cdot 10^{3}$ & 1.41 & 1.4 & 1.44 & $1.69 \cdot 10^{-3}$ \\
\hline $2 m_{T}$ & $0.60 \cdot 10^{3}$ & 1.64 & 0.96 & 1.70 & $1.66 \cdot 10^{-3}$ \\
\hline
\end{tabular}

Table 2. Scale dependence of the SM $\left(\kappa_{t}=1, \kappa_{g}=0\right)$ LO cross sections, $K$-factors and the resulting values of the ratio $\mathcal{R}$, defined in eq. (2.6).

hand, we take the inclusive Higgs production cross section normalized to its SM value

$$
\mu_{\text {incl }}\left(\kappa_{t}, \kappa_{g}\right)=\frac{\sigma_{\text {incl }}\left(\kappa_{t}, \kappa_{g}\right)}{\sigma_{\text {incl }}^{\mathrm{SM}}} \simeq\left(\kappa_{t}+\kappa_{g}\right)^{2}
$$

We assume the large-luminosity LHC scenario with $3 \mathrm{ab}^{-1}$ of data at $14 \mathrm{TeV}$, and therefore we assign to the measurement of $\mu_{\mathrm{incl}}$ a $10 \%$ systematic uncertainty and negligible statistical uncertainty. On the other hand, in order to reduce the theory uncertainty, we consider as boosted observable the ratio

$$
\mathcal{R}\left(\kappa_{t}, \kappa_{g}\right)=\frac{\sigma_{650 \mathrm{GeV}}\left(\kappa_{t}, \kappa_{g}\right) K_{650 \mathrm{GeV}}}{\sigma_{150 \mathrm{GeV}}\left(\kappa_{t}, \kappa_{g}\right) K_{150 \mathrm{GeV}}},
$$

where $K_{p_{T}^{\min }}$ are the QCD $K$-factors for the SM, computed using MCFM-6.6 (process 204). The transverse momentum cuts of $650 \mathrm{GeV}$ and $150 \mathrm{GeV}$ were chosen by means of a rough optimization. The ratio $\mathcal{R}$ is stable under scale variations, as can be seen from table 2 . We remark that presently no exact NLO computation of the SM Higgs $p_{T}$ spectrum is available. The known NLO results, implemented in MCFM-6.6, assume the heavy top approximation and cannot therefore be used for our study, where the full dependence on the top mass is crucial. Nevertheless, multiplying the exact LO cross section times the SM $K$-factor computed in the $m_{t} \rightarrow \infty$ limit, as in eq. (2.6), is the best approximation available at the present time. From this discussion it is clear that an exact NLO computation of the SM Higgs $p_{T}$ spectrum would be very welcome, and we hope that the QCD community will fill this gap in the near future. ${ }^{7}$

We assign a $10 \%$ systematic uncertainty on each of the cross sections that appear in eq. (2.6), and in addition we consider statistical uncertainties on the number of signal events $N_{p_{T}^{\min }}=\sigma_{p_{T}^{\min }}\left(\kappa_{t}, \kappa_{g}\right) K_{p_{T}^{\min }} \epsilon_{\text {tot }} \int \mathcal{L} d t$, where $\int \mathcal{L} d t=3 \mathrm{ab}^{-1}$ is the integrated LHC luminosity. We do not consider any backgrounds in our exploratory study. The two observables $\mu_{\text {incl }}$ and $\mathcal{R}$ are combined via a simple $\chi^{2}$,

$$
\chi^{2}\left(\kappa_{t}, \kappa_{g}\right)=\left(\frac{\mu_{\text {incl }}\left(\kappa_{t}, \kappa_{g}\right)-\mu_{\text {incl }}^{0}}{\delta \mu_{\text {incl }}}\right)^{2}+\left(\frac{\mathcal{R}\left(\kappa_{t}, \kappa_{g}\right)-\mathcal{R}^{0}}{\delta \mathcal{R}}\right)^{2}
$$

where

$$
\frac{\delta \mu_{\text {incl }}}{\mu_{\text {incl }}^{0}}=\delta_{\text {sys }}, \quad \frac{\delta \mathcal{R}}{\mathcal{R}^{0}}=\sqrt{N_{150 \mathrm{GeV}}^{-1}+N_{650 \mathrm{GeV}}^{-1}+2 \delta_{\mathrm{sys}}^{2}}
$$

\footnotetext{
${ }^{7} \mathrm{~A}$ first step in this direction has been made in ref. [39]. For recent progress in the predictions for $h+$ jet, see also refs. [40, 41].
} 
with $\delta_{\text {sys }}=0.1$. The potential of the boosted Higgs measurement to break the degeneracy along the $\kappa_{t}+\kappa_{g}=$ constant direction is shown in figures $2(\mathrm{a})-(\mathrm{d})$. We consider three different assumptions on the observed inclusive signal strength, $\mu_{\text {incl }}^{0}=0.8,1,1.2$, and on the actual value of the $h \bar{t} t$ coupling, $\kappa_{t}^{0}=0.8,1,1.2$. The actual value of $\kappa_{g}$ is then fixed by $\kappa_{g}^{0}=\sqrt{\mu_{\text {incl }}^{0}}-\kappa_{t}^{0}$, and $\mathcal{R}^{0}=\mathcal{R}\left(\kappa_{t}^{0}, \kappa_{g}^{0}\right)$. In figures $2(\mathrm{a})$-(c) we show the $95 \%$ CL contours obtained from the $\chi^{2}$ in eq. (2.7), assuming an integrated luminosity of $3 \mathrm{ab}^{-1}$ at the $14 \mathrm{TeV}$ LHC. The blue, red and black contours correspond to $\kappa_{t}^{0}=0.8,1,1.2$, respectively. The error band obtained considering only inclusive production is shown as well, shaded in gray. It can be clearly seen that including the boosted Higgs measurement allows one to break the degeneracy: for example, assuming a standard inclusive rate (i.e. $\mu_{\text {incl }}^{0}=1$, figure $2(\mathrm{~b})$ ) but a $h \bar{t} t$ coupling deviating by $\pm 20 \%$ from the standard value (black and blue curves, respectively), the SM point can be excluded at approximately $95 \%$ CL. Figure 2(d) shows instead the sensitivity of our results to a variation of the factorization and renormalization scales. The SM is assumed as input $\left(\kappa_{t}^{0}=1\right.$ and $\left.\kappa_{g}^{0}=0\right)$ and the scale $\mu=\mu_{\text {ren }}=\mu_{\text {fact }}$ is set to $m_{T} / 2, m_{T}$ and $2 m_{T}$. Because we employ the ratio of cross sections $\mathcal{R}$, the preferred region in the plane of couplings depends only mildly on the scale choice.

\subsection{Limitations and possible improvements}

Firstly, we should make it clear that the above is only a rough first estimate of the resolving power of the boosted Higgs regime, based purely on signal rates and without considering any backgrounds. Furthermore, the assumption of a $10 \%$ systematic uncertainty on the boosted Higgs cross sections is likely to be optimistic. Secondly, while in this paper we focused on $h \rightarrow \tau \tau$, we wish to mention briefly other a priori interesting choices for the Higgs decay mode. The dominant channel $h \rightarrow b \bar{b}$ seems challenging due to the overwhelming QCD backgrounds. At very large $p_{T}$ the $b$-quarks are collimated, leading in practice to a dijet-like topology; this could provide an interesting application for jet substructure techniques. On the other hand, the decay $h \rightarrow W W$ should provide an attractive alternative to $h \rightarrow \tau \tau$. These issues certainly warrant a more detailed study.

\section{Resolving the top partner spectrum in composite Higgs models}

The degeneracy between the couplings $\kappa_{t}$ and $\kappa_{g}$ assumes a peculiar form in composite Higgs models. It turns out that in the most popular realizations of the Higgs as a composite pseudo-Nambu-Goldstone (pNGB) boson, the combination $\kappa_{t}+\kappa_{g}$ is insensitive to the 'top partners' (the fermionic resonances that are expected to accompany the top) [19-23], as a consequence of an exact cancellation among their contribution to $\kappa_{t}$ and to $\kappa_{g}$. It follows that no indirect information on the resonances can be extracted from the inclusive Higgs rates. To review how this result arises in the well-known 'Minimal Composite Higgs Model 


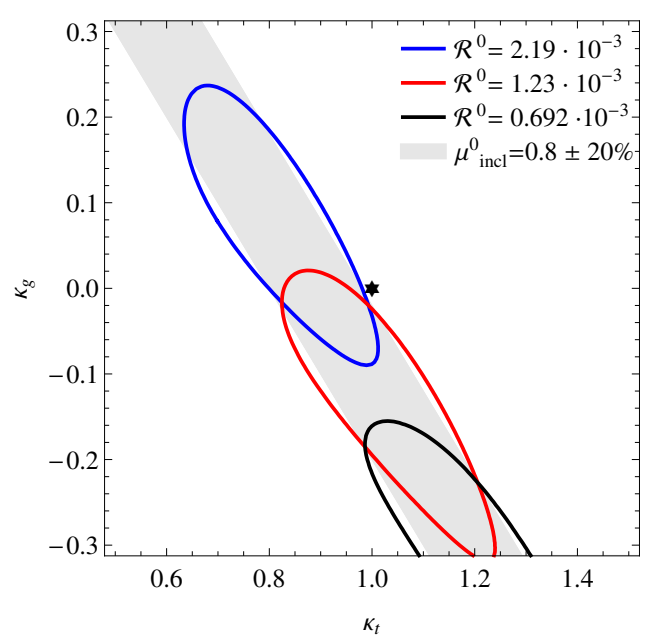

(a) $\mu_{\text {incl }}^{0}=0.8$

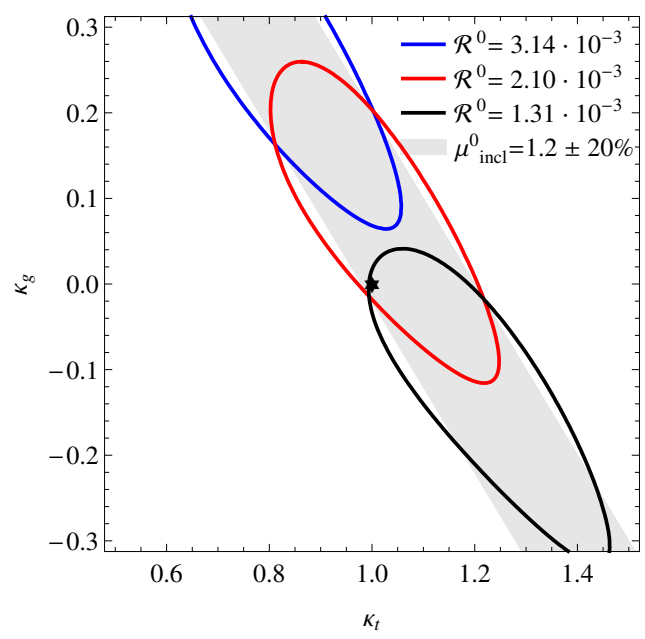

(c) $\mu_{\text {incl }}^{0}=1.2$

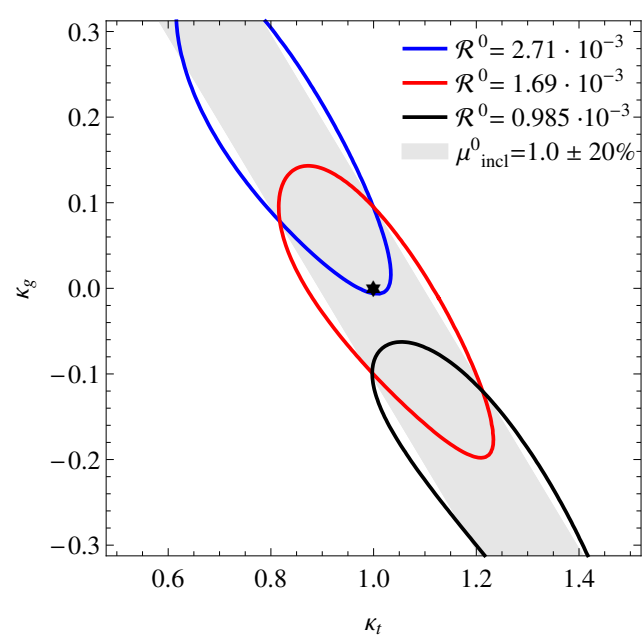

(b) $\mu_{\text {incl }}^{0}=1.0$

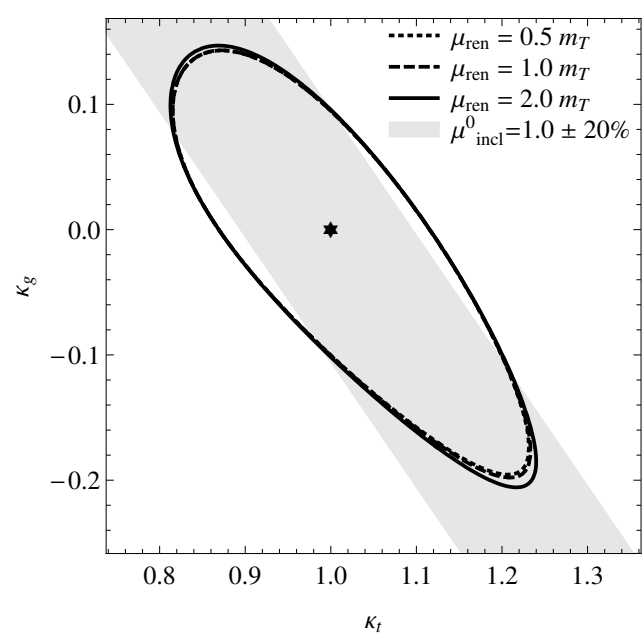

(d) Scale variation

Figure 2. Figures (a)-(c) show the $95 \%$ CL contours obtained from the $\chi^{2}$ in eq. (2.7) for different choices of the actual parameters $\kappa_{t}^{0}$ and $\kappa_{g}^{0}$, or equivalently of $\mu_{\text {incl }}^{0}$ and $\mathcal{R}^{0}$. The colors blue, red and black correspond to $\kappa_{t}^{0}=0.8,1.0$ and 1.2 , respectively, or equivalently to the indicated values of $\mathcal{R}^{0}=\mathcal{R}\left(\kappa_{t}^{0}, \sqrt{\mu_{\text {incl }}^{0}}-\kappa_{t}^{0}\right)$. The gray band is obtained by considering only the inclusive measurement. The SM point is indicated by the black star. Figure (d) shows the variation of the 95\% CL contours for different choices of the renormalization and factorization scale $\mu$. For all plots we assumed an integrated luminosity of $\int \mathcal{L} d t=3 \mathrm{ab}^{-1}$ and $\sqrt{s}=14 \mathrm{TeV}$.

(MCHM)', based on the coset $\mathrm{SO}(5) / \mathrm{SO}(4)$, we first note that ${ }^{8}$

$$
\kappa_{t}+\kappa_{g}=v\left(\frac{\partial}{\partial h} \log \operatorname{det} \mathcal{M}_{t}(h)\right)_{\langle h\rangle},
$$

${ }^{8}$ This formula holds in the natural operator basis for a non-linear $\sigma$-model, see for example ref. [20] for a detailed discussion. The cancellation of the effects of top partners is physical and therefore independent of the basis. 
where $\mathcal{M}_{t}(h)$ is the mass matrix in the top sector. The partial compositeness structure and the pNGB nature of the Higgs imply [23] that the determinant factorizes as $\operatorname{det} \mathcal{M}_{t}(h)=$ $m_{t}^{0}(h) \times \operatorname{det} M_{c}$, where $m_{t}^{0}$ denotes the top mass, ${ }^{9}$ whereas $M_{c}$ is the mass matrix of top partners. We are assuming to be in the field basis where non-derivative interactions of the Goldstone bosons appear only in the linear mixing terms, see eq. (3.2) later for an example. Therefore the matrix $M_{c}$ does not depend on $h$, and its determinant drops out of eq. (3.1). In addition, by means of a spurion argument [21] it can be shown that in models where only one $\mathrm{SO}(4)$ invariant $I_{L R}(h / f)$ can be built out of the embeddings of $t_{L}$ and $t_{R}$, one has $m_{t}^{0}(h) \propto I_{L R}(h / f)$, with $f$ denoting the decay constant of the non-linear sigma model. Thus eq. (3.1) yields simply $\kappa_{t}+\kappa_{g}=f_{g}\left(\xi \equiv v^{2} / f^{2}\right)$, where $f_{g}$ is some function satisfying $f_{g}(\xi \rightarrow 0)=1$. Interestingly, the most viable (and popular) realizations of the MCHM feature only one left-right invariant: for example, the choices $\mathbf{5}_{L}+\mathbf{5}_{R}, \mathbf{1 0}_{L}+\mathbf{1 0}_{R}$ and $\mathbf{1 4}_{L}+\mathbf{1}_{R}$ all lead to $I_{L R}=\sin (2 h / f)$ and therefore to $\kappa_{t}+\kappa_{g}=(1-2 \xi) / \sqrt{1-\xi}$. Furthermore, the insensitivity of the low-energy $h g g$ coupling to the resonances holds also in several Little Higgs models [20]. ${ }^{10}$

\subsection{A simple explicit model}

With the twofold goal of making a simple, concrete example of the above argument and of quantifying the expected size of $\kappa_{g}$ in realistic composite Higgs models, we consider a 'two-site' version of the $\mathrm{MCHM}_{5}$ [42], where one complete $\mathrm{SO}(5)$ multiplet of composite fermions is introduced: the fermion Lagrangian reads

$\mathcal{L}_{f}=i \bar{q}_{L} \not D q_{L}+i \bar{t}_{R} \not D t_{R}+i \bar{\psi} \not D \psi-m_{4} \bar{\psi}_{4} \psi_{4}-m_{1} \bar{\psi}_{1} \psi_{1}-\left(\lambda_{q} \overline{\mathcal{Q}}_{L} U^{T} \psi_{R}+\lambda_{u} \bar{\psi}_{L} U \mathcal{Q}_{R}+\right.$ h.c. $)$

where $\psi=\left(\psi_{4}, \psi_{1}\right)^{T}$ is a complete $\mathbf{5}$ of composite fermions, which decomposes as $\mathbf{5} \sim \mathbf{4} \oplus \mathbf{1}$ under $\mathrm{SO}(4)$, and $\mathcal{Q}_{L, R}$ are the embeddings into incomplete $\mathbf{5} \mathrm{s}$ of $q_{L}$ and $t_{R}$, respectively. The matrix $U$ contains the Goldstone bosons, and the associated decay constant is labeled by $f$. In this setup, the Higgs potential arising from top loops is partially calculable, having the form $V \simeq \alpha \sin ^{2}(h / f)+\beta \sin ^{4}(h / f)$, where $\alpha$ is logarithmically divergent whereas $\beta$ is finite. It follows that the divergence in $\alpha$ can be absorbed by adding a suitable counterterm and fixing the value of the Higgs VEV $v$ as renormalization condition, while the Higgs mass can still be predicted [43]. In order to have a qualitative picture, we set $\xi=0.1$ and scan the parameter space of the model requiring $m_{t}=150 \mathrm{GeV}$ (corresponding roughly to the running top mass at scale $\sim \mathrm{TeV}$ ) and $110 \mathrm{GeV}<m_{h}<140 \mathrm{GeV}$. Figure 3 shows the distribution of the couplings $\kappa_{t}$ and $\kappa_{g}$ versus the mass of the lightest top partner, as obtained from the numerical scan. It is immediate to see 'by eye' that, as predicted by the argument summarized above, the sum $\kappa_{t}+\kappa_{g}$ is the same for all points, being given by $(1-2 \xi) / \sqrt{1-\xi}$. Assuming for example a large $t_{R}$ compositeness, $\kappa_{g}$ has the expression

\footnotetext{
${ }^{9}$ More precisely, $m_{t}^{0}$ is the expression of the top mass when corrections to the wavefunctions of $t_{L, R}$ due to the mixing with top partners are ignored [23].

${ }^{10}$ Exceptions to this result exist. In particular, the insensitivity to the resonance masses does not hold in versions of the MCHM where multiple left-right invariants appear, such as $\mathrm{MCHM}_{14}$, where $q_{L}$ and $t_{R}$ are embedded into the 14-dimensional representation of $\mathrm{SO}(5)$.
} 


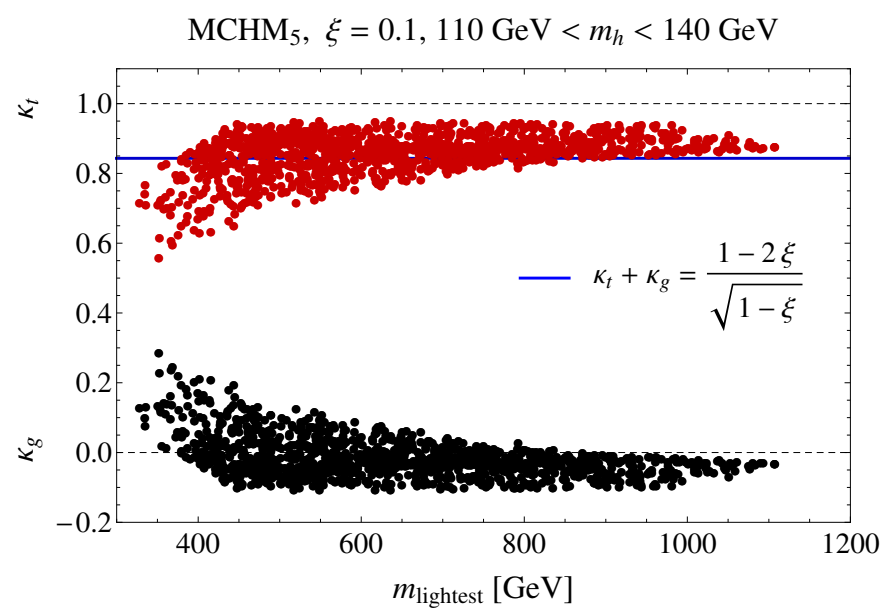

Figure 3. Distribution of the couplings $\kappa_{t}$ and $\kappa_{g}$ versus the mass of the lightest top partner, as obtained from the numerical scan in the two-site $\mathrm{MCHM}_{5}$.

(see ref. [22] for the complete formula)

$$
\kappa_{g}=\xi \sin ^{2} \theta_{R}\left(\frac{m_{1}^{2}-m_{4}^{2}}{m_{4}^{2}}\right)+O\left(\sin ^{2} \theta_{L}\right)
$$

where $\sin \theta_{L, R}=\lambda_{q, u} / \sqrt{m_{4,1}^{2}+\lambda_{q, u}^{2}}$ measures the degree of compositeness of $t_{L, R}$. Combining the full analytical expression of $\kappa_{g}$ [22] with the approximate formula for the Higgs mass [43]

$$
m_{h}^{2} \simeq \frac{N_{c}}{\pi^{2}} \frac{m_{t}^{2}}{f^{2}} \frac{m_{T}^{2} m_{\tilde{T}}^{2}}{m_{T}^{2}-m_{\tilde{T}}^{2}} \log \frac{m_{T}^{2}}{m_{\tilde{T}}^{2}} \quad\left(m_{T, \tilde{T}}=\sqrt{m_{4,1}^{2}+\lambda_{q, u}^{2}}\right)
$$

we find that 'large' values of $\kappa_{g},\left|\kappa_{g}\right|>\xi$, are possible only in the presence of a very light resonance, $m_{\text {lightest }} \ll 1 \mathrm{TeV}$. This is confirmed by the numerical scan: the points yielding $\left|\kappa_{g}\right|>\xi$ have a resonance lighter than $500 \mathrm{GeV}$.

Combining the above discussion with the results of section 2, and in particular with figure 2, it is clear that boosted Higgs production can reveal the presence of light top partners, which would remain otherwise 'hidden' in the measurement of the inclusive rate. ${ }^{11}$ Thus the large- $p_{T}$ Higgs production can usefully complement the information coming from direct searches for the fermionic resonances.

\subsection{Validity of the effective theory approach}

In our analysis of the $\mathrm{MCHM}_{5}$ we have assumed the validity of the Effective Field Theory (EFT) description of new physics states, and computed the coefficient $\kappa_{g}$ by integrating out the top partners. However, given a transverse momentum cut $p_{T}>p_{T}^{\min }$, we naively expect the EFT to break down if there is at least one resonance with mass $M<p_{T}^{\min }$. Since we defined the very boosted region by $p_{T}^{\min }=650 \mathrm{GeV}$, we expect the EFT to be inaccurate for the spectra which feature a top partner with $M \lesssim 650 \mathrm{GeV}$. This is quantified in figure 4 ,

\footnotetext{
${ }^{11}$ See ref. [44] for a recent study of $p p \rightarrow h+$ jet within the context of composite Higgs models.
} 

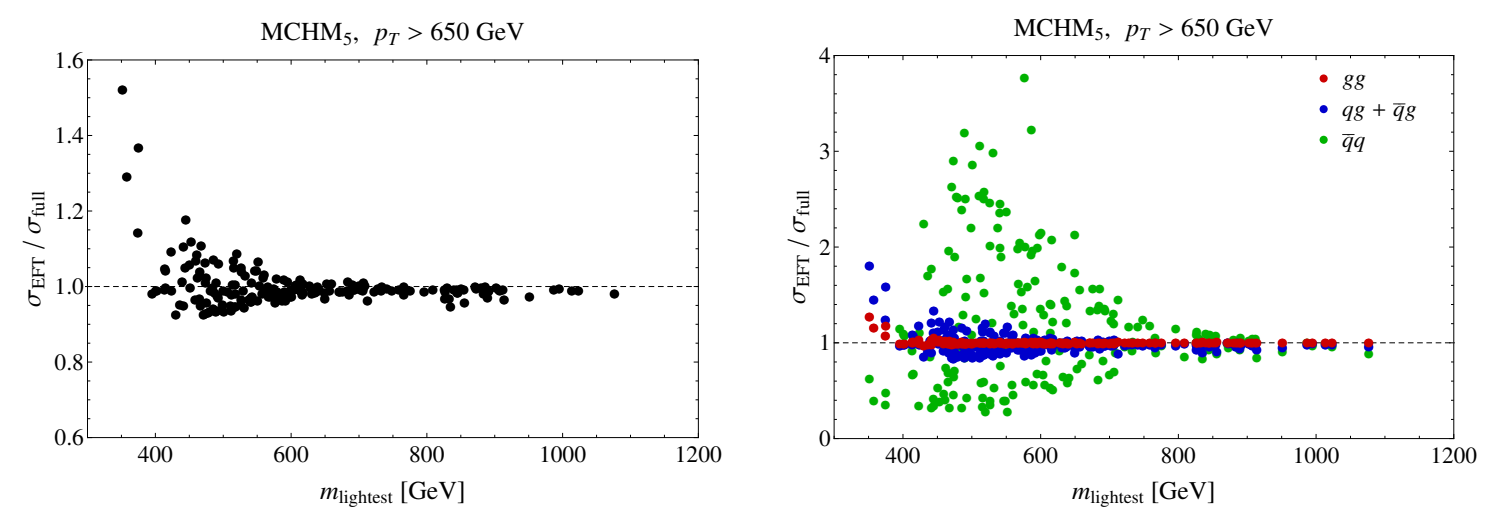

Figure 4. Ratio of the boosted Higgs cross section computed within the effective theory to the exact cross section computed retaining the complete form factors, versus the mass of the lightest top partner, for a sample set of points in the parameter space of $\mathrm{MCHM}_{5}$. A transverse momentum cut $p_{T}>650 \mathrm{GeV}$ is applied. The left panel shows the total cross section $p p \rightarrow h+$ jet, whereas the right panel shows the three partonic channels $g g, q g, q \bar{q} \rightarrow h+$ jet individually.

where we compare the boosted cross section computed in the EFT approximation to the full result obtained by keeping the complete loop form factors. We find that the EFT is accurate within $10 \%$ for top partners as light as $500 \mathrm{GeV}$. We also show a comparison of the EFT and exact cross sections for each partonic initial state, namely for $g g, q g$ and $q \bar{q}$. For the $g g$ channel the EFT is accurate within a few percent, whereas for $q g$ the approximation works within $20 \%$ for resonances above $500 \mathrm{GeV}$. Recalling from table 1 that for $p_{T}>650 \mathrm{GeV}$ the $g g$ and $q g$ channels each make up about $50 \%$ of the total cross section, this yields the already mentioned $10 \%$ overall accuracy. By contrast, the breakdown of the EFT in the $q \bar{q}$ channel is striking, as already noticed in ref. [39]. This effect is however numerically small, since the $q \bar{q}$ channel only contributes about $1 \div 2 \%$ of the total cross section.

\section{Resolving the stop spectrum/mixing in supersymmetric models}

In supersymmetric models the $g g \rightarrow h$ rate is sensitive to loop contributions of stops and sbottoms, affecting both the inclusive [45-49] and the boosted cross section [50-53]. In the following we focus on the Minimal Supersymmetric SM (MSSM) and assume that additional $D$ - or $F$-term contributions help lift the Higgs mass into the phenomenologically allowed range. We further assume that the associated beyond-the-MSSM degrees of freedom do not significantly change the properties of the lightest $C P$-even Higgs. Besides the supersymmetric partners of the SM diagrams of figure 1, new topologies sensitive to the stop trilinear term $A_{t}$ can contribute to the cross section, see figure 5 . The inclusive signal strength is given by [54]

$$
\frac{\Gamma(g g \rightarrow h)}{\Gamma(g g \rightarrow h)_{\mathrm{SM}}}=\left(1+\Delta_{t}\right)^{2}
$$



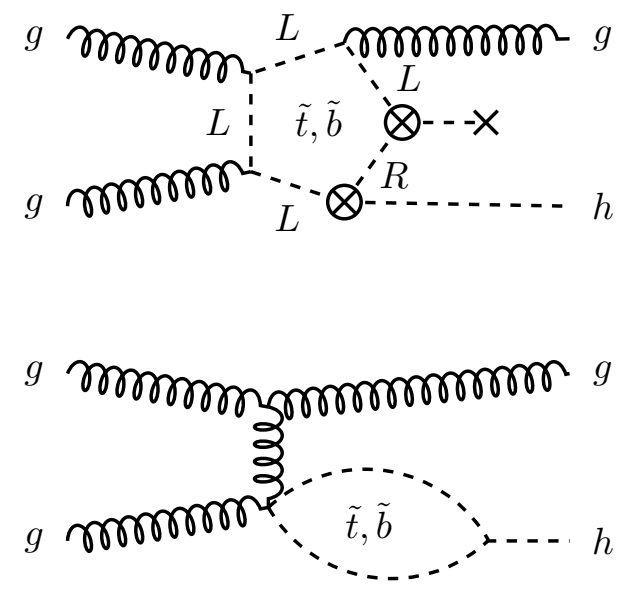
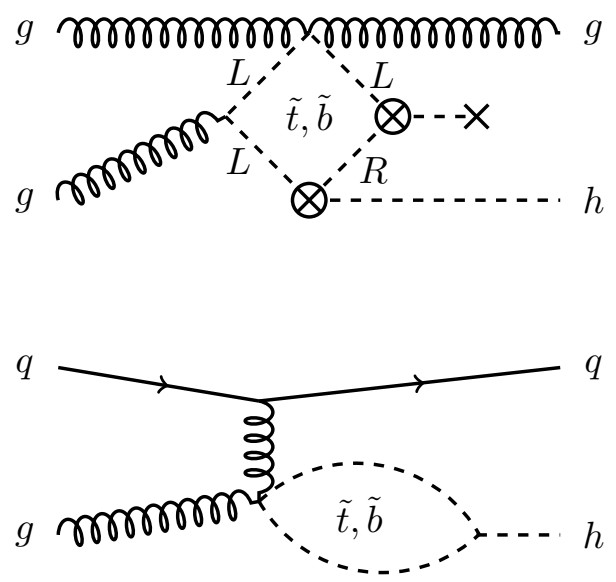

Figure 5. Example Feynman diagrams for $p p \rightarrow h+$ jet involving supersymmetric particles. In addition, there are diagrams like those in figure 1 , but with the quarks in the loops replaced by squarks.

with

$$
\Delta_{t} \approx \frac{m_{t}^{2}}{4}\left(\frac{1}{m_{\tilde{t}_{1}}^{2}}+\frac{1}{m_{\tilde{t}_{2}}^{2}}-\frac{\left(A_{t}-\mu / \tan \beta\right)^{2}}{m_{\tilde{t}_{1}}^{2} m_{\tilde{t}_{2}}^{2}}\right),
$$

We work in the limit where the pseudoscalar Higgs decouples, see e.g. refs. [24, 55], and we have ignored small $D$-term contributions. As in composite Higgs models, we encounter a flat direction: for large enough $A_{t}$, the stop contribution to the inclusive rate can be made to vanish. In figure 6 we show this cancellation as a function of the stop masses and of $A_{t}$. This condition requires large $A_{t}$ and one might worry about vacuum stability, see for instance ref. [56]. A large $A_{t}$ leads to a large trilinear scalar coupling $\propto h A_{t} \tilde{t}_{L} \tilde{t}_{R}^{*}$. If all three fields aquire vacuum expectation values, the potential can have a deep charge- and color-breaking minimum, separated only by a relatively low potential barrier from the usual electroweak vacuum. A rough but conservative estimate of the vacuum stability condition is given by $[57,58]$

$$
A_{t}^{2}+3 \mu^{2}<a \cdot\left(m_{\tilde{t}_{1}}^{2}+m_{\tilde{t}_{2}}^{2}\right)
$$

with $a \approx 3$. This vacuum stability condition is shown in figure 6 , colored in grey. We further identify the regions of parameter space which are excluded because the soft masses $M_{Q_{3}}, M_{U_{3}}$ are not real (orange).

Direct limits from ATLAS and CMS significantly constrain the allowed parameter space. An exhaustive re-analysis of the spectra and decays of all possible light and mixed stops is, however, beyond the scope of our paper. While current experimental searches exclude a significant part of the stop parameter space, these limits soften considerably for larger LSP masses, close to kinematic degeneracies and in the presence of more complicated decay chains, or in the absence of the traditional missing $E_{T}$ signatures (see e.g. refs. [5962]). In particular, light stops with $m_{\tilde{t}_{1}}-m_{\tilde{\chi}_{0}} \approx m_{t}$ are still compatible with data [63-74]. It is therefore interesting to ask whether we can be sensitive to light and mixed stops 

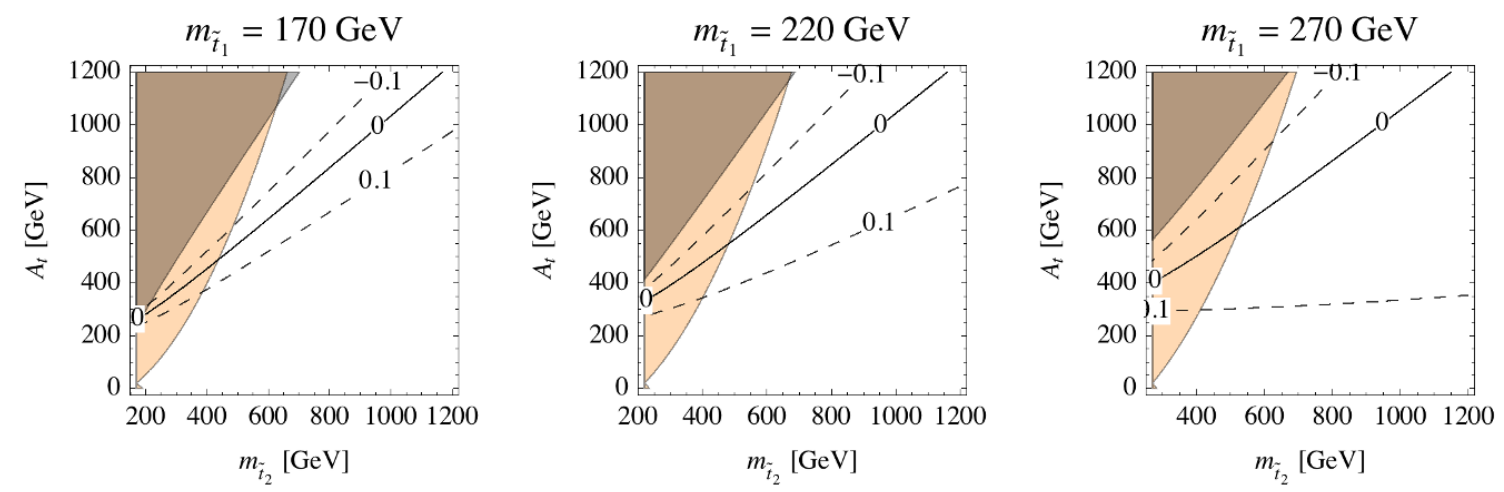

Figure 6. We show the relative deviation of the $g g \rightarrow h$ amplitude from the SM value due to the stop contribution, $\Delta_{t}=0, \pm 0.1$ (solid and dashed lines, respectively). In addition we plot the parameter space excluded because the soft masses are not real (orange) and an estimate of the vacuum stability constraint (grey).

independently of the assumptions on their decays and even if their contribution cancels in the inclusive rate.

We calculated the relevant Feynman diagrams involving the stops using FeynArts3.7 [75, 76] and FormCalc-8.0 [33]. We made use of the MSTW 2008 LO PDFs [32] and of LHAPDF-5.8.9 [77]. The factorization and renormalization scales were set to the minimum transverse mass determined by the cut on $p_{T}, \mu=\sqrt{m_{h}^{2}+p_{T}^{\min 2}}$. In figure 7 we show the dependence of the boosted cross-section on the minimum $p_{T}$ of the Higgs. We plot three benchmark points which would lead to a vanishing contribution to the inclusive crosssection $\left(P_{1}, P_{2}, P_{3}\right)$ and one point $\left(P_{4}\right)$ which shares the same stop masses with $\left(P_{3}\right)$ but has a vanishing $A_{t}$. The latter is included to illustrate that the $A_{t}$-independent terms are mostly responsible for the departure from the point-like approximation. We find that the $p_{T}$ dependence resolves the cancellation of the supersymmetric contribution to the inclusive rate. Naturally, since we are showing the case where the leading effect is completely canceled, the overall size of the contribution will be small. In the less extreme case of a partial cancellation the high- $p_{T}$ measurement could be important to retain sensitivity to the stop contribution.

In conclusion, we find that a precision measurement of the high- $p_{T}$ Higgs cross-section can break the degeneracy present in the inclusive rate and render a 'stealth' stop contribution visible.

\section{Conclusions and outlook}

Gluon fusion is the dominant Higgs production mechanism at the LHC. The current measurements cannot resolve the infrared contribution to the amplitude generated by the top loop from the ultraviolet contribution due to possible new physics, such as the top partners needed to render the Higgs naturally light. The 'traditional' approach to break this degeneracy is provided by the $p p \rightarrow t \bar{t} h$ process, where a direct measurement of the $h \bar{t} t$ 


\begin{tabular}{|r|r|r|r|r|}
\hline Point & $m_{\tilde{t}_{1}}[\mathrm{GeV}]$ & $m_{\tilde{t}_{2}}[\mathrm{GeV}]$ & $A_{t}[\mathrm{GeV}]$ & $\Delta_{t}$ \\
\hline$P_{1}$ & 171 & 440 & 490 & 0.0026 \\
$P_{2}$ & 192 & 1224 & 1220 & 0.013 \\
$P_{3}$ & 226 & 484 & 532 & 0.015 \\
$P_{4}$ & 226 & 484 & 0 & 0.18 \\
\hline
\end{tabular}

Table 3. The benchmark points shown in figure 7 . We set $\tan \beta=10, M_{A^{0}}=500 \mathrm{GeV}, M_{2}=$ $1000 \mathrm{GeV}, \mu=200 \mathrm{GeV}$ and all trilinear couplings to a common value $A_{t}$. The remaining sfermion masses were set to $1 \mathrm{TeV}$ and the mass of the lightest $C P$-even Higgs was set to $125 \mathrm{GeV}$.

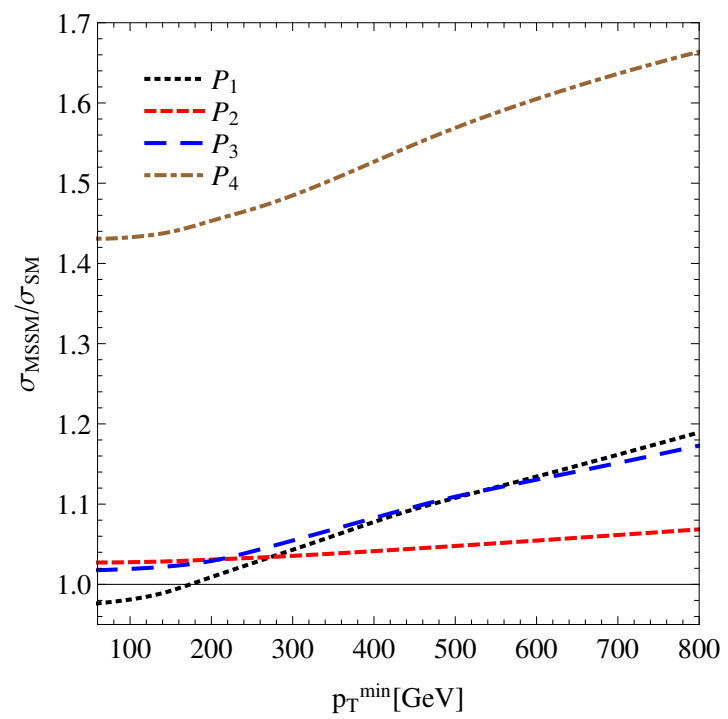

Figure 7. Cross section for boosted Higgs in the MSSM, normalized to the SM value, as a function of the transverse momentum cut $p_{T}^{\min }$. The different lines correspond to the stop masses and values of $A_{t}$ given in table 3 .

coupling can be performed. The objective experimental difficulty of this channel, however, makes it worthwhile to investigate alternatives.

In this paper we considered the production of a boosted Higgs in association with a high- $p_{T}$ jet, where the extra radiation allows one to probe the structure of the top loop and thus to disentangle the IR and UV contributions to the amplitude. The potential of the LHC to resolve the degeneracy was estimated, by using an effective approach to parameterize the new physics. We focused on the decay $h \rightarrow \tau \tau$ and performed an exploratory analysis based only on signal rates.

Subsequently, we discussed the application of our results to two main candidates for a solution to the hierarchy problem, namely partial compositeness at the $\mathrm{TeV}$ scale and natural SUSY. In the former case, the degeneracy between the IR and UV amplitudes for $g g \rightarrow h$ takes the form of a peculiar insensitivity of the inclusive cross section, $\sigma(p p \rightarrow$ $h+X)$, to the presence of light fermionic resonances. In the case of supersymmetry, a large $A_{t}$-term can conspire to make the inclusive rate of SM size even in the presence of 
light stops. We illustrated how, in both scenarios, the boosted Higgs regime can probe the spectrum of top partners (either fermionic or scalar), and thus complement the information coming from direct LHC searches for the resonances.

As a concluding remark, we emphasize that our estimate of the resolving power of the boosted Higgs regime is based only on signal rates, without the inclusion of any backgrounds. As such, it should be regarded as a first, preliminary attempt. More detailed studies are needed in order to reliably compare the reach of the boosted Higgs channel with the expected sensitivity of $p p \rightarrow t \bar{t} h$ and thus to determine the ultimate accuracy attainable by the LHC in the measurement of the coupling of the Higgs to the top quark.

Note added. While this work was nearing completion, ${ }^{12}$ an independent study appeared [81] which has also estimated the sensitivity on the $h \bar{t} t$ coupling determination using the boosted $p p \rightarrow h+$ jet channel, and defined a variable similar to our observable $\mathcal{R}$ to measure the boosted signal.

\section{Acknowledgments}

We thank R. Contino, M. Grazzini, A. Juste, F. Maltoni, A. Pomarol, G. Salam, P. Uwer and L. Vacavant for insightful discussions. We are grateful to M. Spira for correspondence about HIGLU. This research has been partly supported by the European Commission under the ERC Advanced Grant $226371 \mathrm{Mass} T e V$ and the contract PITN-GA-2009-237920 UNILHC. C.G. is supported by the Spanish Ministry MICINN under contract FPA201017747. E.S. was supported in part by the US Department of Energy under grant DE-FG0291ER40674 and by the European Commission under the ERC Advanced Grant 267985 DaMeSyFla. M.S. is supported by the Joachim Herz Stiftung. The work of A.W. was supported in part by the German Science Foundation (DFG) under the Collaborative Research Center (SFB) 676.

\section{A Amplitudes for $p p \rightarrow h+$ jet with $C P$-violating couplings}

In this appendix we collect the analytical expressions of the amplitudes contributing to $p p \rightarrow h+$ jet for $C P$-violating Higgs couplings. We consider first the one-loop amplitudes mediated by the coupling $\tilde{\kappa}_{t}$ in eq. (1.3).

The $g g \rightarrow h g$ amplitude can be expressed in terms of helicity amplitudes $\mathcal{M}_{\lambda_{1} \lambda_{2} \lambda_{3}}$, where the $\lambda_{i}$ denote the helicities of the incoming $(i=1,2)$ and outgoing $(i=3)$ gluons. Out of the eight possible helicity combinations only four are independent and related to the remaining four amplitudes through $\mathcal{M}_{\lambda_{1} \lambda_{2} \lambda_{3}}=\mathcal{M}_{-\lambda_{1}-\lambda_{2}-\lambda_{3}}$. The amplitudes are given

\footnotetext{
${ }^{12}$ For early reports on our results, see the talks by MS at the Spring Conference of the German Physical Society (DPG), Dresden, March 2013; by CG at EPS 2013, Stockholm, July 2013; and by AW at SUSY 2013, Trieste, August 2013 [78-80].
} 
by $^{13}$

$$
\begin{aligned}
& \mathcal{M}_{+++}(s, t, u)=\mathcal{N} F_{1}(s, t, u), \\
& \mathcal{M}_{++-}(s, t, u)=\mathcal{N} F_{1}(s, u, t), \\
& \mathcal{M}_{-+-}(s, t, u)=\mathcal{N} F_{2}(s, t, u), \\
& \mathcal{M}_{-++}(s, t, u)=\mathcal{N} F_{3}(s, t, u),
\end{aligned}
$$

where the form factors $F_{i}$ are defined as

$$
\begin{aligned}
F_{1}(s, t, u) & =\sqrt{\frac{t}{s u}}[G(s, t)-G(s, u)+G(t, u)], \\
F_{2}(s, t, u) & =-\frac{m_{h}^{2}}{\sqrt{s t u}}[G(s, t)+G(s, u)+G(t, u)], \\
F_{3}(s, t, u) & =\sqrt{\frac{s}{t u}}[G(s, t)+G(s, u)-G(t, u)], \\
G(x, y) & =x y D_{0}(x, y)+2 x C_{0}(y)+2 y C_{0}(x),
\end{aligned}
$$

with $C_{0}(x) \equiv C_{0}\left(0, x, m_{h}^{2}, m_{t}^{2}, m_{t}^{2}, m_{t}^{2}\right)$ and $D_{0}(x, y) \equiv D_{0}\left(0,0,0, m_{h}^{2}, x, y, m_{t}^{2}, m_{t}^{2}, m_{t}^{2}, m_{t}^{2}\right)$, where the scalar integrals are given in the conventions of ref. [33]. The common factor $\mathcal{N}$ reads

$$
\mathcal{N}=\frac{\sqrt{3} \alpha_{s}^{3 / 2} m_{t}^{2} \tilde{\kappa}_{t}}{\sqrt{\pi} v}
$$

The unaveraged cross section is then given by

$$
\sum_{\text {pol. }}|\mathcal{M}(g g \rightarrow h g)|^{2}=\sum_{\lambda_{1} \lambda_{2} \lambda_{3}}\left|\mathcal{M}_{\lambda_{1} \lambda_{2} \lambda_{3}}\right|^{2}
$$

Similarly, the squared matrix element for $q \bar{q} \rightarrow h g$ can be expressed in helicity amplitudes $\mathcal{M}_{\lambda_{1} \lambda_{2} \lambda_{3}}^{q \bar{q}}$ where $\lambda_{1,2}$ now denote the polarization of the incoming quark and anti-quark, respectively, and $\lambda_{3}$ the helicity of the outgoing gluon. The four non-zero combinations are related via

$$
\mathcal{M}_{R L+}^{q \bar{q}}(s, t, u)=-\mathcal{M}_{L R-}^{q \bar{q}}(s, t, u)=-M_{R L_{-}}^{q \bar{q}}(s, u, t)=\mathcal{M}_{L R+}^{q \bar{q}}(s, u, t)
$$

and given by

$$
\mathcal{M}_{R L+}^{q \bar{q}}(s, t, u)=-\frac{2 \sqrt{2}}{\sqrt{3 s}} \mathcal{N} t C_{0}(s) .
$$

The unaveraged squared matrix element for the unpolarized cross section is then given by

$$
\sum_{\text {pol. }}\left|\mathcal{M}_{q \bar{q}}(s, t, u)\right|^{2}=\sum_{\lambda_{1} \lambda_{2} \lambda_{3}}\left|\mathcal{M}_{\lambda_{1} \lambda_{2} \lambda_{3}}^{q \bar{q}}(s, t, u)\right|^{2}=\frac{16}{3} \mathcal{N}^{2} \frac{t^{2}+u^{2}}{s}\left|C_{0}(s)\right|^{2} .
$$

\footnotetext{
${ }^{13}$ We define $s=\left(p_{1}+p_{2}\right)^{2}, t=\left(p_{1}-p_{3}\right)^{2}$ and $u=\left(p_{1}-p_{4}\right)^{2}$, where $p_{1,2}$ are the momenta of the ingoing gluons, $p_{3}$ is the momentum of the outgoing gluon and $p_{4}$ the momentum of the Higgs. Conservation of momentum is expressed as $p_{1}+p_{2}=p_{3}+p_{4}$.
} 
Finally, the matrix elements squared for the processes $q g \rightarrow q h$ and $\bar{q} g \rightarrow \bar{q} h$ are obtained via the permutations

$$
\sum_{\text {pol. }}\left|\mathcal{M}_{q g}(s, t, u)\right|^{2}=-\sum_{\text {pol. }}\left|\mathcal{M}_{q \bar{q}}(u, t, s)\right|^{2}, \quad \sum_{\text {pol. }}\left|\mathcal{M}_{\bar{q} g}(s, t, u)\right|^{2}=-\sum_{\text {pol. }}\left|\mathcal{M}_{q \bar{q}}(t, s, u)\right|^{2}
$$

respectively.

For a large mass of the fermion running in the loops, $m_{t}^{2} \gg s,-t,-u, m_{h}^{2}$, we can expand the scalar functions $C_{0}(x)$ and $D_{0}(x, y)$ in powers of $1 / m_{t}^{2}$. Keeping only the leading terms we have

$$
C_{0}(x) \rightarrow-\frac{1}{2 m_{t}^{2}}, \quad D_{0}(x, y) \rightarrow \frac{1}{6 m_{t}^{4}}
$$

In this limit the amplitudes $\mathcal{M}_{g g, q \bar{q}}$ simplify greatly: they are independent of $m_{t}$, and equal to the tree-level amplitudes computed using the effective coupling proportional to $\tilde{\kappa}_{g}$ in eq. (1.3) (the equality holds for $\tilde{\kappa}_{t}=\tilde{\kappa}_{g}$ ). The amplitudes squared and summed over polarizations take the simple form

$$
\sum_{\text {pol. }}\left|\mathcal{M}_{g g}\right|^{2} \rightarrow \frac{3 g_{s}^{6} \tilde{\kappa}_{t}^{2}}{8 \pi^{4} v^{2}} \frac{s^{4}+t^{4}+u^{4}+m_{h}^{8}}{s t u}, \quad \sum_{\text {pol. }}\left|\mathcal{M}_{q \bar{q}}\right|^{2} \rightarrow \frac{g_{s}^{6} \tilde{\kappa}_{t}^{2}}{16 \pi^{4} v^{2}} \frac{t^{2}+u^{2}}{s} .
$$

Open Access. This article is distributed under the terms of the Creative Commons Attribution License (CC-BY 4.0), which permits any use, distribution and reproduction in any medium, provided the original author(s) and source are credited.

\section{References}

[1] ATLAS collaboration, Observation of a new particle in the search for the Standard Model Higgs boson with the ATLAS detector at the LHC, Phys. Lett. B 716 (2012) 1 [arXiv: 1207.7214] [INSPIRE].

[2] CMS collaboration, Observation of a new boson at a mass of $125 \mathrm{GeV}$ with the CMS experiment at the LHC, Phys. Lett. B 716 (2012) 30 [arXiv:1207.7235] [INSPIRE].

[3] W. Buchmüller and D. Wyler, Effective Lagrangian Analysis of New Interactions and Flavor Conservation, Nucl. Phys. B 268 (1986) 621 [InSPIRE].

[4] B. Grzadkowski, M. Iskrzynski, M. Misiak and J. Rosiek, Dimension-Six Terms in the Standard Model Lagrangian, JHEP 10 (2010) 085 [arXiv: 1008.4884] [INSPIRE].

[5] G.F. Giudice, C. Grojean, A. Pomarol and R. Rattazzi, The Strongly-Interacting Light Higgs, JHEP 06 (2007) 045 [hep-ph/0703164] [INSPIRE].

[6] R. Contino, M. Ghezzi, C. Grojean, M. Muhlleitner and M. Spira, Effective Lagrangian for a light Higgs-like scalar, JHEP 07 (2013) 035 [arXiv: 1303.3876] [INSPIRE].

[7] J. Elias-Miro, J.R. Espinosa, E. Masso and A. Pomarol, Higgs windows to new physics through $D=6$ operators: constraints and one-loop anomalous dimensions, JHEP 11 (2013) 066 [arXiv: 1308.1879] [INSPIRE].

[8] A. Pomarol and F. Riva, Towards the Ultimate SM Fit to Close in on Higgs Physics, JHEP 01 (2014) 151 [arXiv: 1308.2803] [INSPIRE]. 
[9] R.V. Harlander and T. Neumann, Probing the nature of the Higgs-gluon coupling, Phys. Rev. D 88 (2013) 074015 [arXiv: 1308.2225] [INSPIRE].

[10] J.R. Ellis, M.K. Gaillard and D.V. Nanopoulos, A Phenomenological Profile of the Higgs Boson, Nucl. Phys. B 106 (1976) 292 [inSPIRE].

[11] M.A. Shifman, A.I. Vainshtein, M.B. Voloshin and V.I. Zakharov, Low-Energy Theorems for Higgs Boson Couplings to Photons, Sov. J. Nucl. Phys. 30 (1979) 711 [inSPIRE].

[12] M. Gillioz, R. Grober, C. Grojean, M. Muhlleitner and E. Salvioni, Higgs Low-Energy Theorem (and its corrections) in Composite Models, JHEP 10 (2012) 004 [arXiv: 1206.7120] [INSPIRE].

[13] CMS collaboration, Projected Performance of an Upgraded CMS Detector at the LHC and HL-LHC: Contribution to the Snowmass Process, arXiv:1307.7135 [INSPIRE].

[14] ATLAS collaboration, Physics at a High-Luminosity LHC with ATLAS, arXiv:1307.7292 [INSPIRE].

[15] H. Baer et al., The International Linear Collider Technical Design Report - Volume 2: Physics, arXiv:1306.6352 [INSPIRE].

[16] TLeP Design Study Working Group collaboration, M. Bicer et al., First Look at the Physics Case of TLEP, JHEP 01 (2014) 164 [arXiv:1308.6176] [INSPIRE].

[17] R.V. Harlander, S. Liebler and T. Zirke, Higgs Strahlung at the Large Hadron Collider in the 2-Higgs-Doublet Model, JHEP 02 (2014) 023 [arXiv:1307.8122] [INSPIRE].

[18] C. Englert, M. McCullough and M. Spannowsky, Gluon-initiated associated production boosts Higgs physics, Phys. Rev. D 89 (2014) 013013 [arXiv:1310.4828] [INSPIRE].

[19] A. Falkowski, Pseudo-goldstone Higgs production via gluon fusion, Phys. Rev. D 77 (2008) 055018 [arXiv: 0711.0828] [INSPIRE].

[20] I. Low and A. Vichi, On the production of a composite Higgs boson, Phys. Rev. D 84 (2011) 045019 [arXiv: 1010.2753 ] [INSPIRE].

[21] A. Azatov and J. Galloway, Light Custodians and Higgs Physics in Composite Models, Phys. Rev. D 85 (2012) 055013 [arXiv:1110.5646] [INSPIRE].

[22] C. Delaunay, C. Grojean and G. Perez, Modified Higgs Physics from Composite Light Flavors, JHEP 09 (2013) 090 [arXiv: 1303.5701] [INSPIRE].

[23] M. Montull, F. Riva, E. Salvioni and R. Torre, Higgs Couplings in Composite Models, Phys. Rev. D 88 (2013) 095006 [arXiv: 1308.0559] [INSPIRE].

[24] A. Delgado, G.F. Giudice, G. Isidori, M. Pierini and A. Strumia, The light stop window, Eur. Phys. J. C 73 (2013) 2370 [arXiv:1212.6847] [InSPIRE].

[25] U. Langenegger, M. Spira, A. Starodumov and P. Trueb, SM and MSSM Higgs Boson Production: Spectra at large transverse Momentum, JHEP 06 (2006) 035 [hep-ph/0604156] [INSPIRE].

[26] C. Arnesen, I.Z. Rothstein and J. Zupan, Smoking Guns for On-Shell New Physics at the LHC, Phys. Rev. Lett. 103 (2009) 151801 [arXiv:0809.1429] [INSPIRE].

[27] E. Bagnaschi, G. Degrassi, P. Slavich and A. Vicini, Higgs production via gluon fusion in the POWHEG approach in the SM and in the MSSM, JHEP 02 (2012) 088 [arXiv:1111.2854] [INSPIRE]. 
[28] R.K. Ellis, I. Hinchliffe, M. Soldate and J.J. van der Bij, Higgs Decay to tau+ tau-: A Possible Signature of Intermediate Mass Higgs Bosons at the SSC, Nucl. Phys. B 297 (1988) 221 [INSPIRE].

[29] U. Baur and E.W.N. Glover, Higgs Boson Production at Large Transverse Momentum in Hadronic Collisions, Nucl. Phys. B 339 (1990) 38 [inSPIRE].

[30] H. Mantler and M. Wiesemann, Top- and bottom-mass effects in hadronic Higgs production at small transverse momenta through LO+NLL, Eur. Phys. J. C 73 (2013) 2467 [arXiv: 1210.8263] [INSPIRE].

[31] M. Grazzini and H. Sargsyan, Heavy-quark mass effects in Higgs boson production at the LHC, JHEP 09 (2013) 129 [arXiv:1306.4581] [INSPIRE].

[32] A.D. Martin, W.J. Stirling, R.S. Thorne and G. Watt, Parton distributions for the LHC, Eur. Phys. J. C 63 (2009) 189 [arXiv:0901.0002] [InSPIRE].

[33] T. Hahn and M. Pérez-Victoria, Automatized one loop calculations in four-dimensions and D-dimensions, Comput. Phys. Commun. 118 (1999) 153 [hep-ph/9807565] [InSPIRE].

[34] J.M. Campbell, R.K. Ellis and C. Williams, MCFM web page http://mcfm.fnal.gov/.

[35] M. Spira, HIGLU: A program for the calculation of the total Higgs production cross-section at hadron colliders via gluon fusion including QCD corrections, hep-ph/9510347 [INSPIRE].

[36] R.P. Kauffman and W. Schaffer, QCD corrections to production of Higgs pseudoscalars, Phys. Rev. D 49 (1994) 551 [hep-ph/9305279] [INSPIRE].

[37] A. Katz, M. Son and B. Tweedie, Ditau-Jet Tagging and Boosted Higgses from a Multi-TeV Resonance, Phys. Rev. D 83 (2011) 114033 [arXiv: 1011.4523] [INSPIRE].

[38] LhC Higgs Cross Section Working Group collaboration, S. Heinemeyer et al., Handbook of LHC Higgs Cross Sections: 3. Higgs Properties, arXiv:1307.1347 [INSPIRE].

[39] R.V. Harlander, T. Neumann, K.J. Ozeren and M. Wiesemann, Top-mass effects in differential Higgs production through gluon fusion at order $\alpha_{s}^{4}$, JHEP 08 (2012) 139 [arXiv: 1206.0157] [INSPIRE].

[40] R. Boughezal, F. Caola, K. Melnikov, F. Petriello and M. Schulze, Higgs boson production in association with a jet at next-to-next-to-leading order in perturbative QCD, JHEP 06 (2013) 072 [arXiv: 1302.6216] [INSPIRE].

[41] S. Hoeche, F. Krauss and M. Schonherr, Uncertainties in MEPS@NLO calculations of $h+$ jets, arXiv: 1401.7971 [INSPIRE].

[42] G. Panico and A. Wulzer, The Discrete Composite Higgs Model, JHEP 09 (2011) 135 [arXiv:1106.2719] [INSPIRE].

[43] O. Matsedonskyi, G. Panico and A. Wulzer, Light Top Partners for a Light Composite Higgs, JHEP 01 (2013) 164 [arXiv: 1204.6333] [INSPIRE].

[44] A. Banfi, A. Martin and V. Sanz, Probing top-partners in Higgs + jets, arXiv:1308.4771 [INSPIRE].

[45] Z. Kunszt and F. Zwirner, Testing the Higgs sector of the minimal supersymmetric standard model at large hadron colliders, Nucl. Phys. B 385 (1992) 3 [hep-ph/9203223] [InSPIRE].

[46] V.D. Barger, M.S. Berger, A.L. Stange and R.J.N. Phillips, Supersymmetric Higgs boson hadroproduction and decays including radiative corrections, Phys. Rev. D 45 (1992) 4128 [INSPIRE]. 
[47] H. Baer, M. Bisset, C. Kao and X. Tata, Observability of gamma gamma decays of Higgs bosons from supersymmetry at hadron supercolliders, Phys. Rev. D 46 (1992) 1067 [INSPIRE].

[48] J.F. Gunion and L.H. Orr, Detecting the Higgs bosons of the minimal supersymmetric model, Phys. Rev. D 46 (1992) 2052 [INSPIRE].

[49] J.F. Gunion, H.E. Haber and C. Kao, Searching for the CP odd Higgs boson of the minimal supersymmetric model at hadron supercolliders, Phys. Rev. D 46 (1992) 2907 [INSPIRE].

[50] A. Djouadi, M. Spira and P.M. Zerwas, Production of Higgs bosons in proton colliders: QCD corrections, Phys. Lett. B 264 (1991) 440 [InSPIRE].

[51] M. Spira, A. Djouadi, D. Graudenz and P.M. Zerwas, Higgs boson production at the LHC, Nucl. Phys. B 453 (1995) 17 [hep-ph/9504378] [INSPIRE].

[52] S. Dawson, Radiative corrections to Higgs boson production, Nucl. Phys. B 359 (1991) 283 [INSPIRE].

[53] D. Graudenz, M. Spira and P.M. Zerwas, QCD corrections to Higgs boson production at proton proton colliders, Phys. Rev. Lett. 70 (1993) 1372 [INSPIRE].

[54] H.E. Haber and G.L. Kane, Implications of a Higgs Interpretation of the Zeta (8.3), Nucl. Phys. B 250 (1985) 716 [INSPIRE].

[55] J.R. Espinosa, C. Grojean, V. Sanz and M. Trott, NSUSY fits, JHEP 12 (2012) 077 [arXiv:1207.7355] [INSPIRE].

[56] M. Reece, Vacuum Instabilities with a Wrong-Sign Higgs-Gluon-Gluon Amplitude, New J. Phys. 15 (2013) 043003 [arXiv: 1208.1765] [INSPIRE].

[57] J.A. Casas, A. Lleyda and C. Muñoz, Strong constraints on the parameter space of the MSSM from charge and color breaking minima, Nucl. Phys. B 471 (1996) 3 [hep-ph/9507294] [INSPIRE].

[58] A. Kusenko, P. Langacker and G. Segre, Phase transitions and vacuum tunneling into charge and color breaking minima in the MSSM, Phys. Rev. D 54 (1996) 5824 [hep-ph/9602414] [INSPIRE].

[59] Y. Kats and D. Shih, Light Stop NLSPs at the Tevatron and LHC, JHEP 08 (2011) 049 [arXiv:1106.0030] [INSPIRE].

[60] J. Fan, M. Reece and J.T. Ruderman, A Stealth Supersymmetry Sampler, JHEP 07 (2012) 196 [arXiv: 1201.4875] [INSPIRE].

[61] J. Fan, M. Reece and J.T. Ruderman, Stealth Supersymmetry, JHEP 11 (2011) 012 [arXiv:1105.5135] [INSPIRE].

[62] Y. Bai, A. Katz and B. Tweedie, Pulling Out All the Stops: Searching for RPV SUSY with Stop-Jets, JHEP 01 (2014) 040 [arXiv: 1309.6631] [INSPIRE].

[63] ATLAS collaboration, Search for direct third-generation squark pair production in final states with missing transverse momentum and two b-jets in $\sqrt{s}=8$ TeV pp collisions with the ATLAS detector, JHEP 10 (2013) 189 [arXiv:1308.2631] [INSPIRE].

[64] ATLAS collaboration, Search for pair-produced top squarks decaying into a charm quark and the lightest neutralinos with $20.3 \mathrm{fb}^{-1}$ of pp collisions at $\sqrt{\mathrm{s}}=8 \mathrm{TeV}$ with the ATLAS detector at the LHC, ATLAS-CONF-2013-068 (2013).

[65] ATLAS collaboration, Searches for direct scalar top pair production in final states with two leptons using the stransverse mass variable and a multivariate analysis technique in $\sqrt{s}=8$ TeV pp collisions using $20.3 \mathrm{fb}^{-1}$ of ATLAS data, ATLAS-CONF-2013-065 (2013). 
[66] ATLAS collaboration, Search for strong production of supersymmetric particles in final states with missing transverse momentum and at least three b-jets using $20.1 \mathrm{fb}^{-} 1$ of $\mathrm{pp}$ collisions at $\sqrt{s}=8 \mathrm{TeV}$ with the ATLAS Detector, ATLAS-CONF-2013-061 (2013).

[67] ATLAS collaboration, Search for direct top squark pair production in final states with two leptons in $\sqrt{s}=8 \mathrm{TeV}$ pp collisions using 20 fb ${ }^{-1}$ of ATLAS data., ATLAS-CONF-2013-048 (2013).

[68] ATLAS collaboration, Search for direct top squark pair production in final states with one isolated lepton, jets and missing transverse momentum in sqrts $=8, \mathrm{TeV}$ pp collisions using $21 \mathrm{fb}^{-1}$ of ATLAS data, ATLAS-CONF-2013-037 (2013).

[69] ATLAS collaboration, Search for direct stop pair production in events with a $Z$ boson, b-jets and missing transverse energy with the ATLAS detector using $21 \mathrm{fb}^{-1}$ from proton-proton collision at $\sqrt{s}=8 \mathrm{TeV}$, ATLAS-CONF-2013-025 (2013).

[70] ATLAS collaboration, Search for direct production of the top squark in the all-hadronic ttbar + etmiss final state in $21 \mathrm{fb}-1$ of p-pcollisions at $\sqrt{s}=8 \mathrm{TeV}$ with the ATLAS detector, ATLAS-CONF-2013-024 (2013).

[71] CMS collaboration, Search for top-squark pair production in the single-lepton final state in pp collisions at $\sqrt{s}=8 \mathrm{TeV}$, Eur. Phys. J. C 73 (2013) 2677 [arXiv:1308.1586] [InSPIRE].

[72] CMS collaboration, Search for SUSY Partners of Top and Higgs Using Diphoton Higgs Decays, CMS-PAS-SUS-13-014 (2013).

[73] CMS collaboration, Search for supersymmetry in pp collisions at $\sqrt{s}=8 \mathrm{TeV}$ in events with three leptons and at least one b-tagged jet, CMS-PAS-SUS-13-008 (2013).

[74] CMS collaboration, Search for direct top squark pair production in events with a single isolated lepton, jets and missing transverse energy at $\sqrt{s}=8 \mathrm{TeV}$, CMS-PAS-SUS-12-023 (2012).

[75] T. Hahn, Generating Feynman diagrams and amplitudes with FeynArts 3, Comput. Phys. Commun. 140 (2001) 418 [hep-ph/0012260] [INSPIRE].

[76] T. Hahn and C. Schappacher, The Implementation of the minimal supersymmetric standard model in FeynArts and FormCalc, Comput. Phys. Commun. 143 (2002) 54 [hep-ph/0105349] [INSPIRE].

[77] M.R. Whalley, D. Bourilkov and R.C. Group, The Les Houches accord PDFs (LHAPDF) and LHAGLUE, hep-ph/0508110 [inSPIRE].

[78] M. Schlaffer, Effective direct and indirect top constraints in Higgs production and decay, Slides of the talk at the Spring Conference of the German Physical Society (DPG), March 2013 [https://bib-pubdb1.desy.de/record/153275/files/DESY-2013-00455.pdf]

[79] C. Grojean, The scalar sector of the SM and beyond, Slides of the talk at EPS 2013 [https://indico.cern.ch/getFile.py/access? contribId=946\&sessionId $=34 \&$ resId $=0 \&$ materialId $=$ slides\&confId $=218030]$.

[80] A. Weiler, Colliding Sflavors, Slides of the talk at SUSY 2013 [http://susy2013.ictp.it/lecturenotes/05_Friday/Weiler.pdf].

[81] A. Azatov and A. Paul, Probing Higgs couplings with high $p_{T}$ Higgs production, JHEP 01 (2014) 014 [arXiv: 1309.5273] [INSPIRE]. 